\title{
Correction: Firearm-related experiences and perceptions among United States male veterans: A qualitative interview study
}

\author{
Joseph A. Simonetti, Brooke Dorsey Holliman, Ryan Holliday, Lisa A. Brenner, Lindsey \\ L. Monteith
}

The third author's name is spelled incorrectly. The correct name is: Ryan Holliday. The correct citation is: Simonetti JA, Dorsey Holliman B, Holliday R, Brenner LA, Monteith LL (2020)

Firearm-related experiences and perceptions among United States male veterans: A qualitative interview study. PLoS ONE 15(3): e0230135. https://doi.org/10.1371/journal.pone.0230135

\section{Reference}

1. Simonetti JA, Dorsey Holliman B, Holiday R, Brenner LA, Monteith LL (2020) Firearm-related experiences and perceptions among United States male veterans: A qualitative interview study. PLoS ONE 15(3): e0230135. https://doi.org/10.1371/journal.pone.0230135 PMID: 32155211

\section{G. openaccess}

Citation: Simonetti JA, Dorsey Holliman B, Holliday R, Brenner LA, Monteith LL (2020) Correction: Firearm-related experiences and perceptions among United States male veterans: A qualitative interview study. PLoS ONE 15(4): e0231493. https://doi.org/10.1371/journal.pone.0231493

\section{Published: April 2, 2020}

Copyright: ๑ 2020 Simonetti et al. This is an open access article distributed under the terms of the Creative Commons Attribution License, which permits unrestricted use, distribution, and reproduction in any medium, provided the original author and source are credited. 